\title{
Dynamic Error Concealment Algorithm for Multiview Coding Using Lost MBs Sizes and Adaptively Selected Candidates MBs
}

\author{
Mohamed Ebian1, Mohamed El-Sharkawy 2 and Salwa El-Ramly 3 \\ 1,3 Electronics and Communications Department, Faculty of Engineering, Ain Shams university ,Egypt \\ 2Electronics and Communications Engineering Department, Egypt-Japan University of Science and Technology \\ (E-JUST), Borg El-Arab, Alexandria, Egypt.
}

\begin{abstract}
The H.264/AVC Multi-View Coding (MVC) compresses more than one viewpoint of a scene captured by different cameras. Redundancies between views and frames are used for inter-view and inra-view prediction ,respectively, in encoding and in Error Concealment (EC) in decoding. As MVC has high error sensitivity, EC algorithms are developed to improve the quality of decoded frames. In this paper, efficient multi-view EC algorithm is proposed for intra MBs and inters MBs exploiting the spatial, inter-view and inra-view information of neighboring MBs. For Intra MBs, Spatial-Inter View (SIV) algorithm is proposed. For inter MBs, the proposed algorithm is adaptively changed according to size of lost MB. In addition, the algorithm dynamically candidates MBs according to the current used view in EC operation. Then the algorithm self generates more candidate MBs depending on best matched MB to get higher matched MB. It is shown that the proposed algorithm leads to better subjective quality in addition to higher PSNR values. By applying the proposed algorithm, the gain is improved by up to $12.4 \mathrm{~dB}$ and $0.73 \mathrm{~dB}$ compared with the case of not applying any EC algorithms and the case of applying normal EC algorithm respectively at $22 \%$ Loss Rate (LR).
\end{abstract}

Keywords-Multi view Coding, Spatial and Temporal Error Concealment, Outer Boundary Matching Algorithm.

\section{Introduction}

Multiview Video (MVV) can be described as multiple video streams shot by several cameras around a single scene simultaneously. MVV technology provides the users with 3DTV and makes Free-Viewpoint Television (FFV) services possible. This provides new applications in entertainment, gaming, medicine and art. Therefore, MVV is expected to be the next revolution in TV broadcasting. Using several cameras in capture operation creates new challenges in delivery due to huge bandwidth required and thus necessitating high compression ratios. Multiview Codec (MVC) is proposed as an extension to H.264 to encode/decode multiview videos. The H.264/AVC multi-view coding tilizes the considerable amount of inter-view redundancies between adjacent views for further compression [1]. In MVC, Motion Vectors (MV) are generated from motion compensation between frames in the same view and Disparity Vectors (DV) are generated from disparity compensation between frames of adjacent views as shown in the MVC prediction structure in Fig. 1.

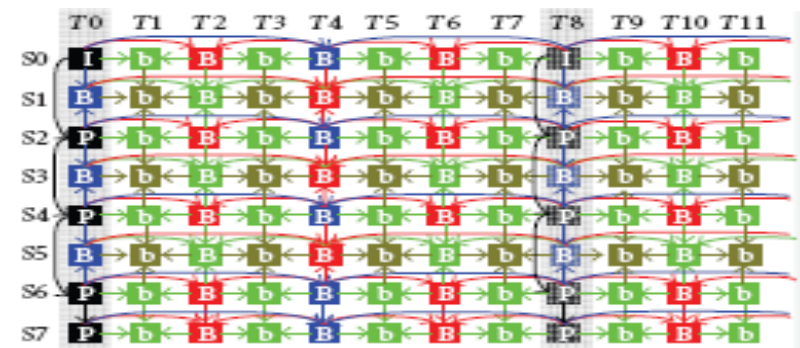

Fig. 1 Prediction Structure of Multi-view Video Coding [1]

In this paper, we propose an adaptive EC algorithm based on lost MB size so the search engine in the reference frames will depend on the lost MB size. The candidate MBs are initially selected according to the view of reference frame. For example, if the reference frame is placed in the right views more candidates in the left side of lost MB is selected in that reference frame.

During algorithm execution, new candidates MBs are generated according to motion direction to get more matched MBs instead of using fixed candidates MBs as in [2]. So the proposed algorithm is considered to exploit the inter-view and intra-view spatio-temporal correlation to conceal MBs to get higher PSNR and higher subjective quality with lower complexity and processing time. The rest of this paper is organized as follows: 
Section 2 is the introduction, Section 3 presents the proposed EC algorithm, Section 4 describes experimental simulation results and Section 5 concludes the paper.

\section{Background}

MVC is of high error sensitivity due to the predictive structure of the MVC. Errors may be propagated through frames in the current view and adjacent view. When packets are lost or error occurred, retransmission of lost packets is a solution but delay constraints will be faced or use of the post processing EC methods at the decoder with no delay cost, no increase in stream transmission bandwidth and no effect in the real-time performance of video transmission. In single view as H.264 code, EC algorithms exploit relations in temporal frames to conceal lost data. But in Multi-view, EC algorithms can profit from the additional information contained in other views of neighboring cameras. The search engine during error concealment of MVC is applied at temporal neighboring frames in current view and in the neighboring frames of other camera views to the left and to the right of the considered view. Then the best matched MB is selected and copied into missing area.

The Boundary Matching Algorithm (BMA) is considered as the basic motion compensation EC technique recommended in H.264/AVC standard for temporal concealment. With MVC, BMA utilizes the MVs plus the DVs of inter-view [3].The selection of the motion/disparity compensated MB is based on the smallest values of Sum of Absolute Differences (SAD).

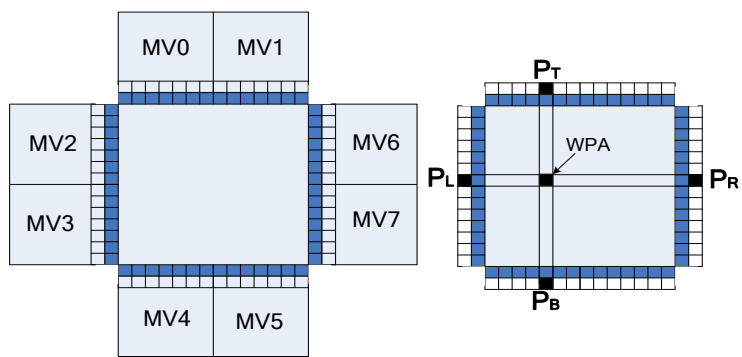

Fig. 2 -Out Boundary Matching Algorithm (left) \& Weighted Average Interpolation (right) [3]

Another EC technique called the Outer BMA (OBMA). The OBMA is generated from the BMA but it gives the differences between the two pixels wide outer boundary of the replacing MB and the same external boundary of the lost corrupted MB as shown in the left part of Fig. 2. OBMA offers better concealment performance than BMA with the same complexity of calculations [4].

\section{Proposed Mvc Error Concealment Algorithm}

The block diagram of the proposed MVC concealment technique is shown in Fig.. 3. The algorithm first checks if the lost MB is intra to apply SIV algorithm. In SIV algorithm, spatial EC called Weighted Pixel Average (WPA) is applied first. The WPA values are obtained inversely proportional to the distance between the reference pixel and the interpolated pixel, as shown in the right part of Fig..2. After applying WPA, DVs are calculated using pixels inside the lost MB and pixels surrounding lost MB position in other views to enhance the initially concealed Intra MB. In SIV algorithm, no temporal EC is applied as the intra MBs has no temporal relations with other frames in same view. So the intra MBs are concealed using spatial information in the same frame and using disparity information in reference frames in the other views.

If the damaged $\mathrm{MB}$ is inter $\mathrm{MB}$, the algorithm determines the reference frames in all views. The reference frames may be located in the same views or in other views. The proposed algorithm applies proposed search engine over all reference frames.

The candidate MBs used in EC algorithm are adaptively selected according to the view of the current reference frame being processed as following:

- If the view of the current reference frame is the same views of lost MB then the candidate MBs are horizontal, vertical and diagonal MBs.

- If the view of the current reference frame is the left view of lost MB view so the candidate MBs are surrounding neighboring MBs (4 neighboring, 4 corner) plus more right neighboring MBs.

- If the view of the current reference frame is the right view of lost MB view then the candidate MBs are surrounding neighboring MBs (4 neighboring, 4 corners) plus more left neighboring MBs. 


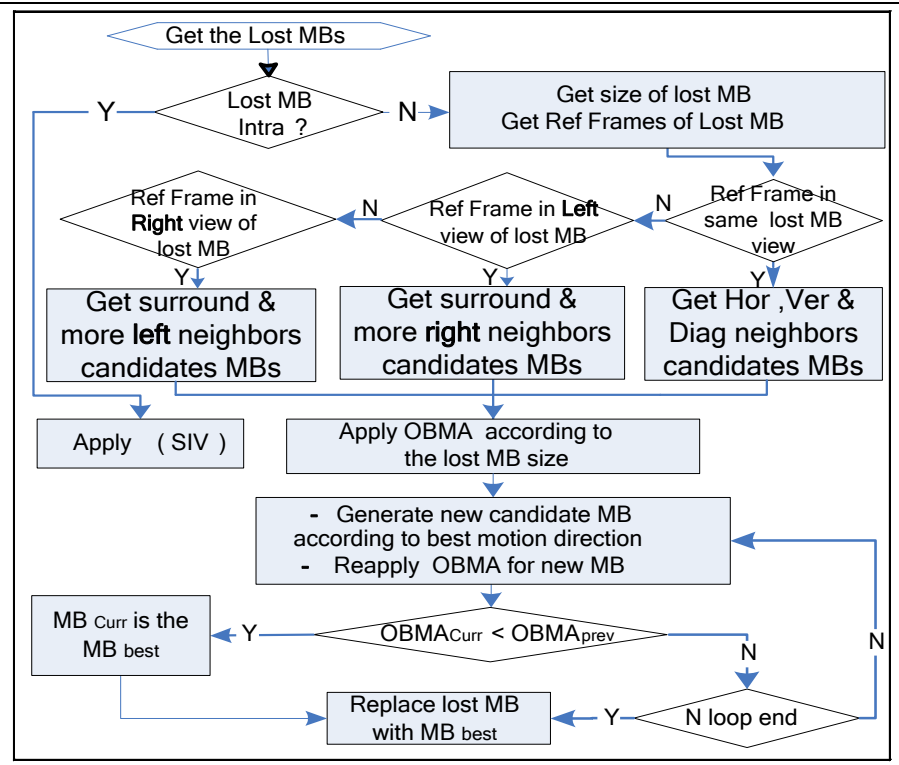

Fig. 3 The proposed algorithm

The selection for more left candidate MBs at right view and right candidate MBs at left view can be explained from Fig. 4. Assume lost MB is placed in middle view (current view). For the left view, the location of lost $\mathrm{MB}$ is placed at the right side of left view. As a result, there is more probability to find more matched MBs for the lost one. So more right candidate MBs are selected for the proposed search algorithm. For the right view, the location of lost $\mathrm{MB}$ is placed at the left side of right view. As a result, there is more probability to find more matched MBs for the lost one. So more left candidate MBs are selected for the proposed search algorithm. The adaptive selection of candidate MBs saves the processing time instead of increasing initial candidate MBs numbers.

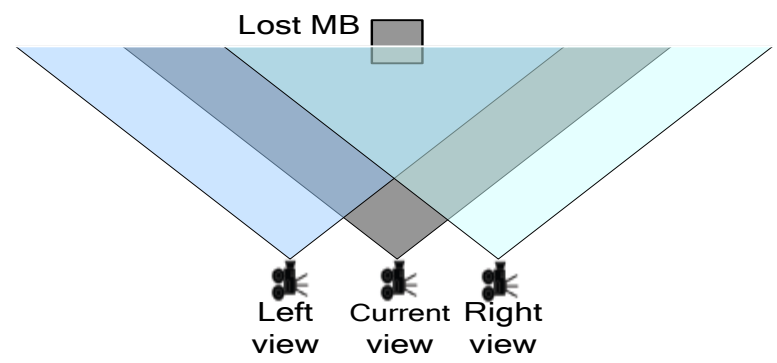

Fig. 4 Location Of Lost MB With Respect To Adjacent Left and Right Views Fig. 5.

The modes (sizes) of lost MBs may be one of the four modes: $16 \times 16,16 \times 8,8 \times 16, \& 8 \times 8$ as shown in

- Mode 1 (16x16): The set of concealed MVs candidates for block 0 is $\{\mathrm{V} 1, \mathrm{~V} 2, . ., \mathrm{V} 8\}$ where $\mathrm{V} 1, \mathrm{~V} 2, \ldots, \mathrm{V} 8$ are referred to all MVs located around lost MB.

- Mode 2 (16x8): The sets of concealed MV candidates for blocks 0 and 1 are $\{$ MV1, MV2, MV3, MV7 $\}$ \& $\{$ MV4, MV5, MV6, MV8\}, respectively.

- Mode 3 (8x16): The sets of MV candidates for blocks 0 and 1 are $\{$ MV1, MV3, MV4, MV5 $\} \&\{$ MV2, MV6, MV7, MV8\}, respectively.

- Mode 4 (8x8): The sets of MV candidates for blocks 0, 1, $2 \& 3$ are $\{$ MV1, MV3\}, \{MV2, MV7\}, \{MV4, MV5\} \& \{MV6, MV8\}, respectively.

After selecting the most suitable partition type for the lost MB, each partition of the lost MB is concealed by applying proposed search algorithm using the first candidate set of MBs depended on current reference frame being processed.

The proposed algorithm divides the candidate $\mathrm{MB}$ into portions with size equal to the lost MB size. Then OBMA is applied between lost MB and each partition in the candidate MB. For example, if lost MB is $8 \times 16$ so that there are two sub MBs (left and right) of size 8x16 are searched in each candidate MB and so for other MBs types till getting the best one with the lowest OBMA value. For better MBs replacement, a self generated candidate MB algorithm is proposed. This algorithm uses the best selected MB direction as a step to 
get more candidate MBs in the same motion direction. With each step, if the OBMA value of new candidate MB is lower than previous one so this new candidate MB is selected as best matched MB and so on till getting best MB through the selected loop number $\mathrm{N}$ as shown in Fig. 3.

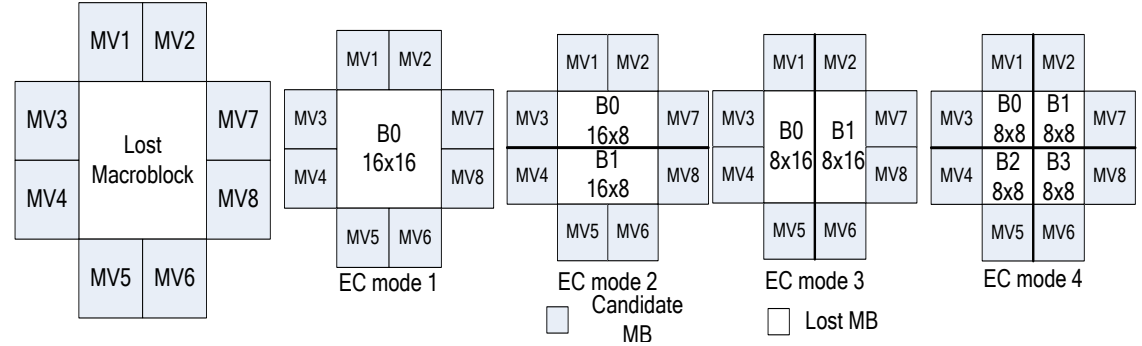

Fig. 5 MV Candidates For Lost MB And Four EC Modes

\section{Simulation And Results}

The proposed algorithm is applied to Joint MVC reference software [5] for 50 frames of ballroom and exit sequences of $640 \times 480$ frame size and $30 \mathrm{~Hz}$ frame rate. An error mask is applied for multiview stream to destroy MBs to get Loss Rate (LR) about 22\% of all MBs as shown in Fig. 6(a). Then, the error mask ratio is decreased to $15 \%, 11 \%$ and $5 \%$.The lost MBs locations are assumed to be known.

Table (1) shows the PSNR results of the reconstructed Ballroom sequence with no error mask, OBMA with fixed size and fixed candidate MBs, not applying any EC algorithms, the proposed algorithm with no adaptive candidate MBs and the proposed algorithm with adaptive candidate MBs. By applying the full proposed algorithm, the PSNR results for ballroom sequence is improved up to $12.4 \mathrm{~dB}$ and $0.73 \mathrm{~dB}$ comparing to applying no EC algorithm and to applying normal OBMA respectively at 22\% PLR.

As shown in table 1, the obtained PSNR results for the full proposed algorithm are higher than the proposed algorithm with fixed candidate MBs with small differences. However, the visual quality for full algorithm is better. Using adaptive candidate MBs saves processing time. If total collection of the candidate MBs are used for all reference frames more processing time is consumed than using specific candidates MBs with each reference frame.

The white dashed circles in Fig. 6(d) indicate visual details improvements with the proposed algorithm than in Fig. 6(b) with normal OBMA. The yellow arrows in Fig. 6(d) indicate visual improvements with using adaptive candidate MBs with each reference frame than in Fig. 6(c) with OBMA and no adaptive candidate MBs. Those improvements are obtained due to making sure that the lost MB is replaced with the same MB size and use of better candidate MBs in the proposed algorithm.

\section{Conclusion}

In this paper, a proposed EC algorithm is suggested to conceal lost MBs in MVC. This algorithm is dynamically changed according to the lost MB size and adaptively selects the candidate MBs according to view contained current reference frame being processed. The proposed algorithm provides considerable better gain in the objective and subjective quality with lower processing time comparing with normal OBMA method.

\section{References}

[1] Y. Chen, Y.K. Wang, K. Ugur, M .M. Hannuksela, J. Lainema, and M. Gabbouj, "The Emerging MVC Standard for 3D Video Services," EURASIP Journal on Advances in Signal Processing, Vol. 2009, Article ID 786015, 13 Pages, 2009

[2] B. W. Micallef, C.J. Debono and R.A. Farrugia, "Performance of Enhanced Error Concealment Techniques in Multi-view Video Coding Systems," in IEEE Int. Conf. on Systems, Signals \& Image Processing, Sarajevo, Bosnia \& Herzegovina, jun. 2011.

[3] B.W. Micallef, and C. J. Debono, "Error Concealment Techniques For Multi-View Video" Proc. of IFIP Wireless Days Conf., Oct. 2010.

[4] Santosh V Chapaneri. Article: Content-Adaptive Refined Error Concealment Schemes for H.264/AVC Video Coding. International Journal of Computer Applications 27(7):36-43, August 2011.

[5] ISO/IEC MPEG \& ITU-T VCEG, "Reference Software - Joint Multiview Video Coding Model (JMVC 5.0.5)," JVT-AE207, June 2009. (JMVC) 8.0 . 


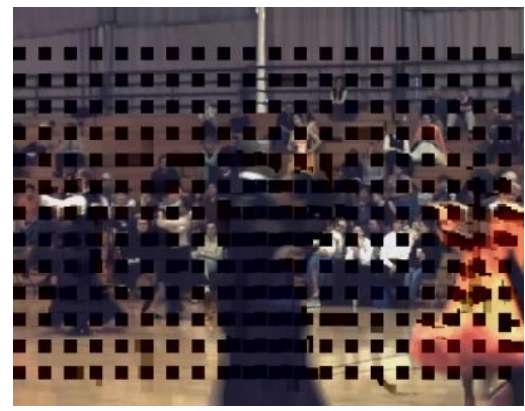

(a)

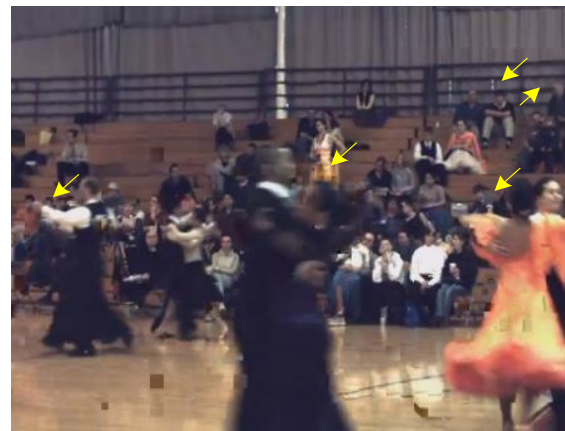

(c)

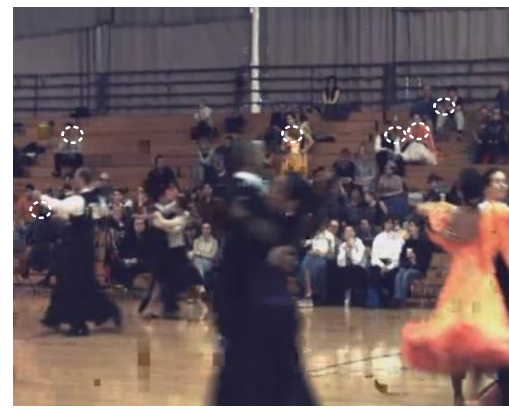

(b)

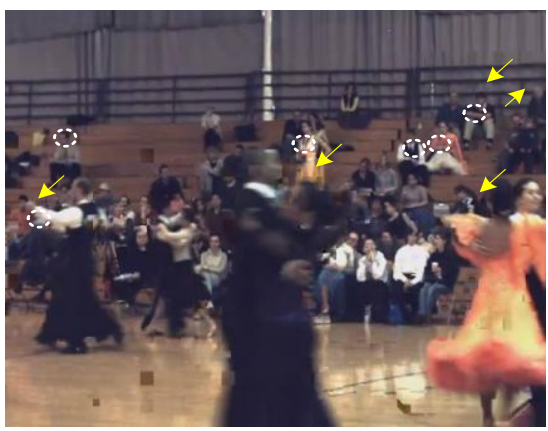

(d)

Fig. 6 Subjective quality comparison for frame 29 of ballroom sequence,(a) Corrupted frame (b) Concealed by normal OBMA

(c) Concealed by proposed OBMA but with out adaptive candidate MBs (d) Concealed by full algorithm

Table 1 PSNR $(\mathrm{dB})$ results for Ballroom test sequence

\begin{tabular}{|c|c|c|c|c|}
\hline \multirow{2}{*}{ Applied EC algorithm } & \multicolumn{4}{|c|}{ Loss Rate } \\
\cline { 2 - 5 } & $\mathbf{5 \%}$ & $\mathbf{1 1 \%}$ & $\mathbf{1 5 \%}$ & $\mathbf{2 2 \%}$ \\
\hline No Error Occur & 33.41 & 33.41 & 33.41 & 33.41 \\
\hline Normal OBMA & 30.39 & 28.8 & 27.94 & 26.49 \\
\hline $\begin{array}{c}\text { Proposed OBMA with no adaptive } \\
\text { candidate MBs }\end{array}$ & 30.50 & 29.10 & 28.50 & 27.14 \\
\hline $\begin{array}{c}\text { Proposed OBMA with adaptive } \\
\text { candidate MBs "Full algorithm" }\end{array}$ & $\mathbf{3 0 . 6 1}$ & $\mathbf{2 9 . 2 4}$ & $\mathbf{2 8 . 5 5}$ & $\mathbf{2 7 . 2 2}$ \\
\hline Error occur without applying EC & 20.75 & 17.82 & 16.48 & 14.75 \\
\hline
\end{tabular}

